\title{
Efeito agudo da vibração sobre o desempenho do agachamento em alta velocidade e salto vertical
}

\author{
Acute effect of whole-body vibration on high velocity squat \\ and jump performance
}

1 Universidade de São Paulo. Escola de Educação Física e Esporte. São Paulo, SP. Brasil

Recebido em 01/10/09 Revisado em 20/03/10 Aprovado em 11/05/10
Resumo - Este estudo teve como objetivo avaliar o efeito agudo da exposição à vibração para o corpo todo sobre a produção de potência de membros inferiores no agachamento convencional e sobre o desempenho do salto vertical. Trinta sujeitos experientes em treinamento de força tiveram avaliados seus desempenhos no agachamento em alta velocidade e no salto vertical com contramovimento, antes e depois de serem expostos a quatro protocolos de vibração de maneira balanceada. As avaliações do agachamento em alta velocidade foram realizadas 3 minutos antes e, 6, 9 e 12 minutos após a exposição à vibração. As avaliações do salto vertical com contramovimento foram realizadas seis minutos antes e, nove e quinze minutos após a exposição à vibração. Nenhuma alteração foi verificada nos desempenhos do agachamento veloz ou do salto vertical em razão da exposição à vibração. No entanto, foi verificado efeito principal do tempo, indicando uma diminuição na altura do salto vertical aos 3 minutos $(-2 \%)$ e aos 15 minutos $(-3,1 \%)$ após o tratamento. Estes resultados sugerem que a exposição à vibração não produz melhoras agudas no desempenho. Contudo, isso não é suficiente para descartar a possibilidade de utilização da vibração para o corpo todo nas rotinas de aquecimento ou de treinamento.

Palavras-chave: Treinamento de potência; Plataforma de vibração; Aquecimento; Salto vertical; Métodos de treinamento.

Abstract - The objective of this study was to assess the acute effect of whole-body vibration (WBV) on power production of the lower limbs during squat exercise and on vertical jump height. The performance of 30 strength-trained subjects was assessed during high velocity squat exercise (HVS) and countermovement vertical jump (CMJ) before and after being submitted to four different vibration protocols in a counterbalanced random manner. The HVS and CMJ assessments were performed 3 min before and 6, 9 and 12 min after the WBV interventions, and 6 min before and 9 and 15 min after the interventions, respectively. The different WBV protocols did not change relative peak or average power production during HVS and CMJ. However, time exerted a main effect, with a decrease in CMJ height at $3 \mathrm{~min}(-2 \%)$ and $15 \mathrm{~min}(-3.1 \%)$ after treatment. These results suggest that the WBV protocols employed in this study do not induce acute improvement in performance. However, this finding does not rule out the application of WBV as a useful strategy for training or warm-up routines.

Key words: Power training; Vibration platform; Warm-up; Vertical jump; Training methods. 


\section{INTRODUÇÃO}

A literatura científica tem demonstrado crescente preocupação com os possíveis efeitos de uma sessão de treinamento de vibração para o corpo todo (TVCT) sobre o desempenho' ${ }^{1-5}$. Acredita-se que a exposição à vibração provoque um aumento transitório da excitabilidade neural central e periférica, o que poderia favorecer o desempenho, nos minutos subsequentes, de tarefas motoras com elevadas demandas de força e potência musculares ${ }^{6,7}$. Em vários destes estudos, o efeito do TVCT foi avaliado em relação ao desempenho do salto vertical ${ }^{1,2,5,8-10}$. Em alguns casos, foram verificadas melhoras transitórias no rendimento, minutos após a exposição ao TVCT ${ }^{1,2,8,11}$. Uma vez que o salto vertical é um importante componente do desempenho em muitas modalidades esportivas, tem sido sugerido aplicar o TVCT nas rotinas de aquecimento a fim de aproveitar seu efeito excitatório durante a competição $0^{8,9}$.

Os efeitos agudos do TVCT sobre a excitabilidade neuromuscular também podem ser explorados em outros contextos ${ }^{12,13}$. Por exemplo, num modelo de treinamento complexo (combinação, na mesma série, de um exercício com carga elevada e, na sequência, um exercício de potência, com carga leve), o TVCT poderia substituir o exercício com carga elevada que precede o exercício de potência. A melhora aguda na potência muscular pode implicar um estímulo de treinamento de maior intensidade, o que pode levar a adaptações crônicas mais acentuadas. Melhoras agudas na potência de membros inferiores após a exposição ao TVCT foram verificadas quando avaliadas no exercício de pressão de pernas ${ }^{3,4}$.

O agachamento é um dos exercícios mais utilizados nos programas de treinamento para o desenvolvimento da potência de membros inferiores $^{14,15}$. Contudo, foi encontrado um único estudo no qual foi feita a avaliação do efeito de uma sessão isolada de TVCT sobre a potência produzida nesse exercício ${ }^{10}$. Este estudo mostrou melhoras similares na potência após a exposição a seis séries de 60 segundos de duração (6 x 60") de TVCT, com as frequências de 20 e $30 \mathrm{~Hz}$, mas não com a frequência de $40 \mathrm{~Hz}$. Isto sugere que os protocolos de aquecimento ou de treinamento com o TVCT devem incluir frequências entre 20 e $30 \mathrm{~Hz}$.

Contudo, o estudo de Silva et al. ${ }^{10}$ deve ser analisado com cautela. Nesse estudo, a melhora da potência foi avaliada imediatamente após a exposição ao TVCT. Assim, não se sabe se alguns minutos após o TVCT ela já haveria se dissipado, o que inviabilizaria sua aplicação num modelo de treino complexo. Além disso, não se sabe se as frequências de TVCT testadas no estudo de da Silva et al. ${ }^{10}$ teriam o mesmo efeito no desempenho do agachamento convencional, uma vez que naquele estudo a potência foi avaliada somente na fase concêntrica do movimento, enquanto o agachamento convencional envolve ações do ciclo alongamento encurtamento (CAE). Baseado nas evidências de efeitos positivos da TVCT no salto vertical, espera-se encontrar o mesmo efeito no agachamento convencional.

Portanto, o objetivo do estudo foi avaliar o efeito agudo da exposição ao TVCT sobre a produção de potência de membros inferiores no agachamento convencional assim como no desempenho do salto vertical, uma vez que ambas as atividades envolvem ações do CAE.

\section{PRODEDIMENTOS METODOLÓGICOS}

\section{Amostra}

Trinta sujeitos do sexo masculino (treinados em força por dois anos) participaram do estudo. Os valores médios para idade (anos), estatura $(\mathrm{cm})$, massa corporal $(\mathrm{kg})$ e percentual de gordura corporal foram: $24,5( \pm 3,3), 173,3( \pm 7,6), 80,5( \pm 12,1)$, e $12,9( \pm 6,1)$, respectivamente. Os sujeitos foram orientados a não se exercitarem 24 horas antes das sessões experimentais. $\mathrm{O}$ estudo foi aprovado pelo comitê de ética em pesquisa da Universidade de São Paulo (Protocolo: 2006/01), tendo todos os sujeitos assinado o termo de consentimento informado.

\section{Desenho experimental}

Os sujeitos compareceram ao laboratório em dez ocasiões. Nas quatro visitas iniciais os sujeitos foram familiarizados aos procedimentos do estudo. Nas seis visitas seguintes, foram submetidos às sessões experimentais.

Para verificar o efeito de uma sessão de TVCT sobre a potência de membros inferiores e sobre o desempenho do salto vertical, todos os sujeitos foram avaliados nos exercícios de agachamento em alta velocidade (AAV) e salto vertical com contramovimento (SVCM) antes e após serem expostos ao TVCT, em quatro diferentes sessões experimentais. Em cada sessao experimental os sujeitos foram expostos a um protocolo diferente de TVCT. O AAV foi avaliado 3 minutos antes, e 6, 9, e 12 minutos após o TVCT. O SVCM foi avaliado 6 minutos antes, e 3 e 15 minutos após o TVCT (Figura 1). Outras duas sessões experimentais fo- 
ram controle, sem exposição ao TVCT. Em uma delas foram realizadas as tarefas de salto vertical e agachamento (Figura 2) e na outra, apenas a tarefa do salto vertical.

Os momentos das avaliações nas sessões controle foram os mesmos das sessões com TVCT. As duas sessões controle tiveram como propósito identificar um possível efeito potencializador do AAV sobre o desempenho do SVCM. As seis sessões experimentais foram realizadas com, pelo menos, $48 \mathrm{~h}$ de intervalo. A ordem das sessões experimentais foi aleatorizada através do procedimento de quadrados latinos adaptado por William ${ }^{16}$.

\section{Procedimentos de familiarização}

As sessões de familiarização foram realizadas em intervalos de 48 horas. No início de todas as sessões de familiarização, os sujeitos realizaram cinco minutos de corrida em esteira ergométrica (Movement ${ }^{\circledR}$, Bruden, São Paulo) a 8,5 km/h, seguidos de cinco minutos de alongamentos de membros inferiores.

Na primeira sessão, avaliou-se a força dinâmica máxima (1RM) de membros inferiores, usando o exercício agachamento paralelo no aparelho Smith $^{17}$. Nas três sessões seguintes, os sujeitos foram familiarizados ao salto com contramovimento (SVCM), ao AAV, e ao protocolo de TVCT, na respectiva ordem.

Antes da familiarização ao SVCM, os sujeitos eram orientados a realizar saltos máximos em cada tentativa, mantendo a mesma posição corporal na decolagem e aterrissagem. Os sujeitos realizaram dez SVCM em cada sessão, com 30 segundos de intervalo entre saltos. Para considerá-los familiarizados ao SVCM, exigiu-se que a altura média atingida nos saltos não variasse mais que 3\% entre diferentes sessões.
Em seguida, para se familiarizarem ao AAV, os sujeitos realizaram três séries de seis repetições. A variabilidade intrassujeitos foi estimada tanto para a amplitude de movimento $(\mathrm{m})$ como para a potência produzida (W), sendo considerados aptos os que atingiam valores inferiores a 3\% de variabilidade. Todos os sujeitos atingiram o índice pré-estabelecido antes da terceira sessão de familiarização ao AAV. O posicionamento dos sujeitos no Smith foi registrado para garantir a reprodutibilidade na avaliação do AAV entre sessões de familiarização e experimentais.

Cinco minutos após a familiarização ao AAV, os sujeitos iniciavam a familiarização ao TVCT. Para isso, eram posicionados na plataforma vibratória na posição de agachamento isométrico com os joelhos e o quadril mantidos a, aproximadamente, $90^{\circ}$ e $110^{\circ}$ de flexão, respectivamente. A posição de apoio dos pés foi registrada para reprodução nas sessões experimentais. Todos os sujeitos realizaram três séries de TVCT, combinando os parâmetros de vibração utilizados nos procedimentos experimentais.

\section{Procedimentos Experimentais}

\section{- Descrição das sessões experimentais}

Ao início de todas as sessões experimentais, os sujeitos realizavam o mesmo procedimento de aquecimento realizado nas sessões de familiarização. Em seguida, tinham seus desempenhos avaliados no AAV e no SVCM antes e depois do TVCT, nos intervalos descritos anteriormente.

\section{- Avaliação da potência de membros inferiores} no AAV

A potência produzida durante o AAV foi avaliada

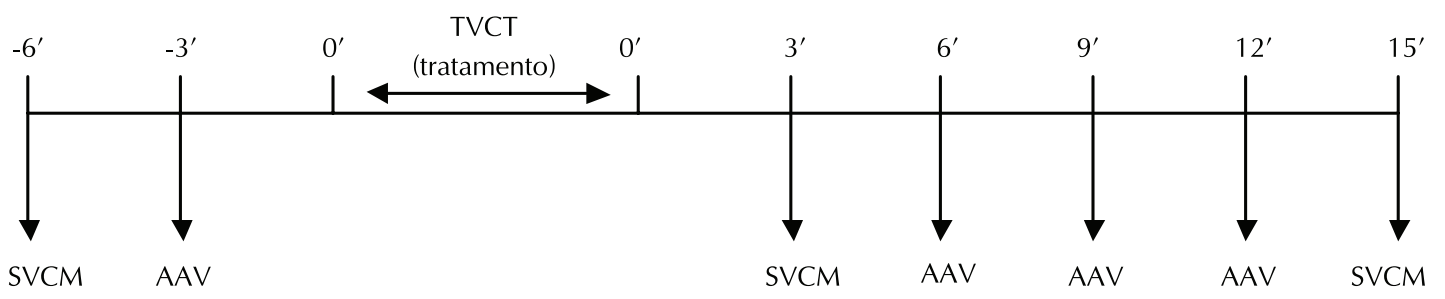

Figura 1. Sessão experimental. TVCT = Protocolo de vibração para o corpo todo.

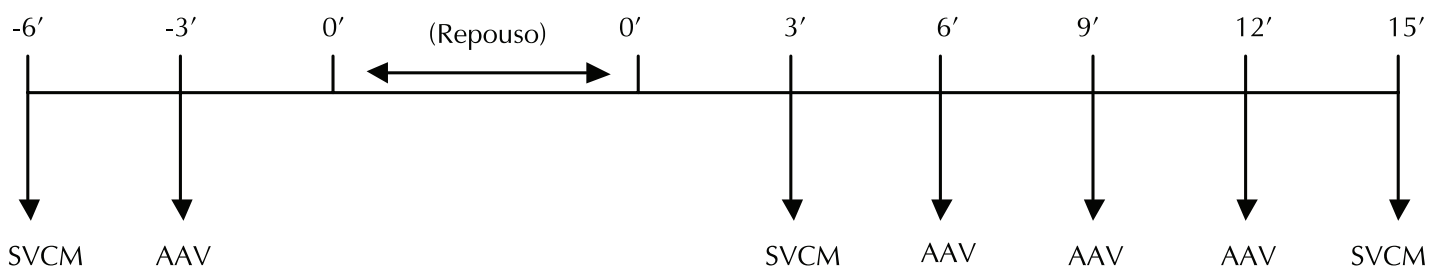

Figura 2. Sessão controle de TVCT sobre o AAV e desempenho do SVCM. 
no Smith (Nakagym ${ }^{\circledR}$, São Paulo, Brasil). Um encoder linear (Peak Power $^{\circledR}$, Cefise, São Paulo, Brasil) foi conectado à barra para registrar a variação de sua posição ao longo das repetições, na frequência de $50 \mathrm{~Hz}$. A técnica de diferenciação finita foi utilizada para a velocidade e a aceleração da barra (coeficiente de variabilidade $<3 \%$ ). Com isso, a força e a potência da barra foram calculadas, utilizando-se procedimentos padronizados ${ }^{18}$. A posição corporal e o posicionamento dos pés foram determinados com fitas métricas fixadas à barra e ao solo, respectivamente. Além disso, foram colocados caixotes de madeira, ajustáveis à altura, abaixo dos sujeitos, para garantir que o deslocamento da barra e a modificação na angulação de flexão dos joelhos fossem constantes de repetição para repetição. Ao comando do avaliador, os sujeitos realizavam seis repetições do AAV com carga de 40\% de 1 RM.

\section{- Avaliação do SVCM}

A avaliação do SVCM foi realizada através de uma plataforma resistiva, conectada a um relógio digital (JumpTest $^{\circledR}$, Belo Horizonte, Brasil). Os sujeitos eram instruídos a manter as mãos na cintura e saltar o mais alto possível em cada tentativa. Além disso, eram orientados a manter a mesma posição corporal durante a decolagem e a aterrissagem do salto. Em cada avaliação, os sujeitos realizaram três SVCM com intervalos de 15 segundos.

\section{- Protocolos de TVCT}

A exposição à vibração foi feita através de uma plataforma vibratória (PowerPlate ${ }^{\circledR}$, Holanda). Durante a exposição à vibração, os sujeitos permaneciam em posição de agachamento isométrico, com os joelhos e o quadril flexionados a, aproximadamente, $90^{\circ}$ e $110^{\circ}$ de flexão, respectivamente, e as mãos apoiadas na cintura. Nessa posição realizavam-se três séries de TVCT com cada um dos seguintes protocolos experimentais: a) 30 segundos $/ 30 \mathrm{~Hz}$; b) 60 segundos $/ 30 \mathrm{~Hz}$; c) 30 segundos $/ 40 \mathrm{~Hz}$; d) 60 segundos $/ 40 \mathrm{~Hz}$. Uma vez que não está claro o efeito da amplitude de vibração sobre o desempenho ${ }^{19}$, nesse estudo, a amplitude de vibração foi mantida entre 4-6 mm para todos os protocolos. Entre as séries de vibração, respeitou-se um intervalo de 60 segundos de repouso.

\section{Análise dos dados}

O salto de melhor desempenho foi usado para verificar o efeito agudo do TVCT sobre o desempenho do SVCM. Uma rotina construída no programa Visual Basic ${ }^{\circledR}$ foi usada para inferir as potências pico e média produzidas na fase concêntrica do AAV. Ambas as variáveis foram calculadas, usando as três repetições com as potências médias mais altas produzidas em cada série do AAV. $\mathrm{O}$ coeficiente de correlação intraclasse (ICC) entre os pré-testes, para o SVCM e para a potência média relativa no $\mathrm{AAV}$, foram 0,98 e 0,95, respectivamente.

\section{Análise Estatística}

Os dados foram analisados quanto à normalidade e presença de observações extremas gráfica e quantitativamente (i.e. Shapiro Wilk). Um modelo misto, tendo condição experimental (5 níveis) e tempo (4 níveis) como fatores fixos, e sujeitos como fatores aleatórios, foi usado para testar a existência de interações para as variáveis potência pico e potência média concêntricas. Outro modelo misto, tendo condição experimental (6 níveis) e tempo (3 níveis) como fatores fixos, e sujeitos como fatores aleatórios, foi usado para testar as diferenças na altura do salto. Utilizou-se um ajuste por Tukey para comparações post-hoc $(\mathrm{p}<0,05)$. As análises estatísticas foram realizadas no pacote estatístico SAS 8.2 e os dados foram apresentados como média \pm desvio padrão.

\section{RESULTADOS}

Os diferentes protocolos de TVCT não modificaram as potências relativas pico e média (Tabelas 1 e 2).

Tabela 1. Potência pico relativa (Média $\pm D P)(W / k g)$ produzida na fase concêntrica do AAV.

\begin{tabular}{lccccc}
\hline & $\mathrm{CON}_{(\mathrm{SVCM}+\mathrm{AaV})}$ & $30 \mathrm{~s} / 30 \mathrm{~Hz}$ & $30 \mathrm{~s} / 40 \mathrm{~Hz}$ & $60 \mathrm{~s} / 30 \mathrm{~Hz}$ & $60 \mathrm{~s} / 40 \mathrm{~Hz}$ \\
\hline Pré 3' $^{\prime}$ & $25,6 \pm 6,3$ & $26,9 \pm 6,9$ & $27,1 \pm 7,5$ & $26,5 \pm 7,0$ & $27,6 \pm 7,3$ \\
Pós 6' & $25,3 \pm 6,1$ & $27,1 \pm 7,5$ & $26,5 \pm 5,1$ & $27,8 \pm 9,5$ & $26,0 \pm 6,1$ \\
Pós 9' & $25,8 \pm 7,0$ & $28,0 \pm 10,2$ & $26,2 \pm 6,3$ & $27,8 \pm 11,4$ & $25,9 \pm 5,8$ \\
Pós 12' & $27,3 \pm 9,2$ & $26,5 \pm 6,1$ & $27,1 \pm 6,9$ & $25,9 \pm 6,0$ & $26,8 \pm 6,7$ \\
\hline
\end{tabular}

$\mathrm{CON}_{(\mathrm{SVCM}+\mathrm{AAV})}=$ Sessão controle para os efeitos do TVCT sobre o agachamento em alta velocidade com $40 \% 1 \mathrm{RM}$ e sobre o desempenho do SVCM. 


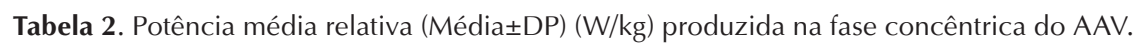

\begin{tabular}{lrrrrr}
\hline & $\mathrm{CON}_{(\mathrm{SVCM}+\mathrm{AAV})}$ & \multicolumn{1}{c}{$30 \mathrm{~s} / 30 \mathrm{~Hz}$} & $30 \mathrm{~s} / 40 \mathrm{~Hz}$ & $60 \mathrm{~s} / 30 \mathrm{~Hz}$ & $60 \mathrm{~s} / 40 \mathrm{~Hz}$ \\
\hline Pré 3' & $8,1 \pm 1,6$ & $8,5 \pm 1,9$ & $8,2 \pm 1,6$ & $8,4 \pm 1,7$ & $8,5 \pm 1,6$ \\
Pós 6' & $8,2 \pm 1,6$ & $8,4 \pm 1,8$ & $8,3 \pm 1,6$ & $8,5 \pm 2,0$ & $8,3 \pm 1,6$ \\
Pós 9' & $8,2 \pm 1,8$ & $8,5 \pm 2,0$ & $8,4 \pm 1,6$ & $8,5 \pm 2,0$ & $8,3 \pm 1,6$ \\
Pós 12' & $8,5 \pm 2,0$ & $8,5 \pm 1,8$ & $8,5 \pm 1,7$ & $8,4 \pm 1,7$ & $8,3 \pm 1,7$ \\
\hline
\end{tabular}

Tabela 3. Altura do salto vertical, em cm, com contramovimento (Média \pm DP) antes e após os protocolos de TVCT.

\begin{tabular}{|c|c|c|c|c|c|c|}
\hline & $\mathrm{CON}_{(\mathrm{SVCM})}$ & $\mathrm{CON}_{(\mathrm{SVCM}+\mathrm{AAV})}$ & $30 \mathrm{~s} / 30 \mathrm{~Hz}$ & $30 \mathrm{~s} / 40 \mathrm{~Hz}$ & $60 \mathrm{~s} / 30 \mathrm{~Hz}$ & $60 \mathrm{~s} / 40 \mathrm{~Hz}$ \\
\hline *Pré 6' & $40,4 \pm 6,3$ & $39,7 \pm 6,3$ & $39,9 \pm 5,3$ & $39,4 \pm 7,6$ & $40,4 \pm 6,1$ & $39,6 \pm 4,8$ \\
\hline Pós 3' & $39,2 \pm 5,7$ & $38,9 \pm 5,9$ & $39,5 \pm 5,5$ & $39,8 \pm 6,1$ & $38,8 \pm 6,3$ & $38,4 \pm 5,6$ \\
\hline
\end{tabular}

* Efeito significante do tempo $(p<0,0001)$. Valores de pré-teste foram maiores que os do pós-teste.

$\mathrm{CON}_{(\mathrm{SVCM})}=$ Sessão controle para os efeitos do AAV sobre o desempenho do SVCM.

Verificou-se efeito principal de tempo significante para a altura do salto vertical $(p<0,0001)$, indicando decréscimo de desempenho nas reavaliações (Tabela 3), com valores de -2,0\% e -3,1\% no terceiro e décimo quinto minutos, respectivamente.

\section{DISCUSSÃO}

O objetivo principal desse estudo foi analisar o efeito da vibração sobre a potência produzida no agachamento envolvendo o CAE. Além disso, também foi testada sua influência sobre o desempenho do SVCM. A exposição ao TVCT em 3 séries de 30 ou 60 segundos de duração, combinada com as frequências de 30 ou $40 \mathrm{~Hz}$ (e amplitude de 4-6 mm), não produziu qualquer alteração significante nos desempenhos de ambas as tarefas motoras avaliadas.

A ausência de alterações no desempenho da potência, no presente estudo, está em desacordo com outros estudos que testaram o efeito do TVCT sobre o desempenho da potência de membros inferiores, porém usando um exercício diferente deste estudo. Por exemplo, Bosco et al. ${ }^{3}$ e Bosco et al. ${ }^{4}$ verificaram aumentos significantes na potência produzida no exercício de pressão de pernas, instantes após a exposição ao TVCT (10 x 60") com frequência de $26 \mathrm{~Hz}$ (10 mm e $4 \mathrm{~mm}$, respectivamente). Numa análise precipitada, esses dados sugerem que o exercício empregado na avaliação da potência pode ter influenciado os resultados considerados.

No entanto, da Silva et al. ${ }^{10}$ testaram o efeito de diferentes frequências de vibração sobre a potência produzida no agachamento concêntrico. Também em desacordo com resultados aqui apresentados, esse estudo verificou a melhora significante na potência minutos após o TVCT (6 x 60", 4 mm) com frequências de $20 \mathrm{~Hz}(1,5 \%)$ e $30 \mathrm{~Hz}(4,6 \%)$ e aumento não significante de $1,2 \%$ com a frequência de $40 \mathrm{~Hz}$.

Por outro lado, até o momento, o presente estudo foi o primeiro a testar o efeito do TVCT sobre a potência produzida no agachamento, envolvendo ações musculares do ciclo alongamento encurtamento (CAE), exercício utilizado com muita frequência nos programas de treinamentos de atletas de diversas modalidades esportivas.

Os efeitos agudos da vibração sobre o desempenho são atribuídos às alterações do stiffness (resistência ao alongamento) neuromuscular, que é influenciado pelo aumento da sensibilidade dos fusos musculares ${ }^{20,21}$. Uma vez que o desempenho em ações do CAE depende do stiffness neuromuscular $^{22-24}$, sugere-se que as frequências de vibração testadas no presente estudo $(30 \mathrm{e} 40 \mathrm{~Hz})$ causariam diferentes alterações no agachamento envolvendo CAE, em comparação às observadas no estudo de da Silva et al. ${ }^{10}$, hipótese esta suportada por outras evidências ${ }^{2,10}$.

Cormie et $a .^{8}$ sugeriram haver uma relação entre o tempo total de exposição ao TVCT e magnitude das alterações observadas no desempenho. Esta possibilidade pode contribuir para explicar as diferenças entre os resultados dos estudos de Bosco et al. ${ }^{3,4}$, respectivamente (6x60" e 3x60") e os encontrados no presente estudo, com uma exposição máxima de 3x60". De acordo com Cormie et $\mathrm{al}^{8}$, tempos totais de exposição mais prolongados produziriam aumentos proporcionais no desempenho (Torvinen et al. ${ }^{5}, 4$ minutos de TVCT= 2,2\% aumento na altura do SVCM; Bosco et al. ${ }^{4}, 10 \mathrm{x}$ 60" TVCT = aumento de 3,9\% na altura do SVCM). Porém, é possível que a relação existente entre o tempo de exposição ao TVCT e a magnitude das alterações observadas no desempenho não seja tão eficiente para diferentes tarefas como proposto por 
Cormie et al. ${ }^{8}$. Foi bem demonstrado que o tempo excessivamente prolongado de exposição à vibração de fato leva à fadiga ${ }^{6,11,25}$. Por isso, optamos por protocolos com tempo total de exposição menor que o utilizado por da Silva et al. ${ }^{10}$.

Além do efeito do TVCT sobre o a potência no agachamento, nós também testamos seu efeito sobre o desempenho do SVCM. Não foi verificada alteração no desempenho do SVCM. Contudo, estudos anteriores verificaram aumentos expressivos no desempenho do SVCM após a exposição ao TVCT 3,4,10, permanecendo a questão em aberto. Diferente do ocorrido para o agachamento, parece que o volume total de estimulação não justifica a ausência de melhora no desempenho do SVCM aqui observado, já que Cormie et al..$^{8}$ e Bazett-Jones et al. ${ }^{11}$ verificaram melhoras no desempenho do SVCM após a aplicação do TCVT, por tempo total de 30 e 45 segundos, respectivamente. Em especial, para frequências próximas a $30 \mathrm{~Hz}$, esperava-se verificar melhora, já que vários estudos verificaram melhoras agudas no SVCM com frequências entre $26 \mathrm{~Hz}^{1,3,4}$ e $30 \mathrm{~Hz}^{10}$.

O efeito principal de tempo para o SVCM ( $p<0,0001)$, indicando uma redução na altura do SVCM nas reavaliações aos 3 e 15 minutos (2,0\% e $3,1 \%$, respectivamente), sugere o advento da fadiga, contribuindo assim para mascarar possíveis melhoras no desempenho ${ }^{26}$. Contudo, considera-se esta hipótese bastante improvável. Os sujeitos avaliados no presente estudo reportaram experiência em treinamento de força de, pelo menos, dois anos, sugerindo aptidão para suportar a carga proporcionada pelo TVCT. Além disso, vários estudos envolvendo sujeitos sem experiência em treinamento de força verificaram melhoras agudas no desempenho empregando TVCT com intensidades e durações superiores às empregadas no presente estudo $24,5,10$. Embora seja razoável admitir que a fadiga possa ter prejudicado o desempenho na reavaliação ao $3^{\circ}$ minuto, seu efeito deveria ter sido dissipado na reavaliação ao $15^{\circ}$ minuto ${ }^{26}$. Além disso, a potência produzida no agachamento não sofreu qualquer alteração no tempo, o que afasta a hipótese de ocorrência de fadiga em função do TVCT.

É ainda possível que a ausência de melhora no desempenho tenha sido provocada pela característica do exercício realizado durante a aplicação da vibração. Durante a exposição à vibração, os sujeitos mantiveram-se em posição de agachamento isométrico, com os joelhos flexionados à $90^{\circ}$. Essa posição é mais profunda que as usadas nos estudos que verificaram melhoras agudas na potência ${ }^{10} \mathrm{e}$ no $\mathrm{SVCM}^{1-4,10}$, o que pode causar maior ativação dos músculos reto femoral, vasto lateral e vasto medial, conforme tendência verificada por Roelants et al. ${ }^{27}$. Dessa forma, o aumento da profundidade do agachamento produz maior ativação muscular nos agonistas, porém não nos isquitibiais ${ }^{28}$, o que assume magnitude ainda maior quando associado à vibração.

\section{CONCLUSÃO}

A exposição à vibração não causou alteração na potência avaliada no agachamento convencional ou na altura do salto vertical. É possível que o tempo total de exposição ao TVCT e a profundidade do agachamento realizado durante a exposição ao TVCT tenham contribuído para a ausência de efeito positivo sobre o desempenho. Porém, não se descarta que o TVCT possa ser eficiente nas rotinas de treinamento ou aquecimento pré-competição, se estruturado de forma apropriada.

\section{REFERÊNCIAS BIBLIOGRÁFICAS}

1. Cochrane DJ, Stannard SR. Acute whole body vibration training increases vertical jump and flexibility performance in elite female field hockey players. Br J Sports Med 2005;39(11):860-5.

2. Cardinale M, Lim J. The acute effects of two different whole body vibration frequencies on vertical jump performance. Med Sport 2003;56(4):287-92.

3. Bosco C, Colli R, Introini E, Cardinale M, Tsarpela $\mathrm{O}$, Madella A, et al. Adaptive responses of human skeletal muscle to vibration exposure. Clin Physiol 1999;19(2):183-7.

4. Bosco C, Iacovelli M, Tsarpela O, Cardinale M, Bonifazi M, Tihanyi J, et al. Hormonal responses to whole-body vibration in men. Eur J Appl Physiol 2000;81(6):449-54.

5. Torvinen S, Kannu P, Sievanen H, Jarvinen TA, Pasanen M, Kontulainen S, et al. Effect of a vibration exposure on muscular performance and body balance. Randomized cross-over study. Clin Physiol Funct Imaging 2002;22(2):145-52.

6. Rittweger J, Mutschelknauss M, Felsenberg D. Acute changes in neuromuscular excitability after exhaustive whole body vibration exercise as compared to exhaustion by squatting exercise. Clin Physiol Funct Imaging 2003;23(2):81-6.

7. Cardinale M, Bosco C. The use of vibration as an exercise intervention. Exerc Sport Sci Rev 2003;31(1):3-7.

8. Cormie P, Deane RS, Triplett NT, McBride JM. Acute effects of whole-body vibration on muscle activity, strength, and power. J Strength Cond Res 2006;20(2):257-61.

9. Cochrane DJ, Stannard SR, Walmsely A, Firth EC. The acute effect of vibration exercise on concentric muscular characteristics. J Sci Med Sport 2008;11(6):527-34. 
10. da Silva ME, Nuñes VM, Vaamonde D, Fernandez JM, Poblador MS, Garcia-Manso JM, et al. Effects of different frequencies of whole body vibration on muscular performance. Biol Sport 2006;23(3):267-82.

11. Bazett-Jones DM, Finch HW, Dugan EL. Comparing the effects of various whole-body vibration accelerations on counter-movement jump performance. J Sports Sci Med 2008;7(1):144-50.

12. Bullock N, Martin DT, Ross A, Rosemond CD, Jordan MJ, Marino FE. Acute effect of whole-body vibration on sprint and jumping performance in elite skeleton athletes. J Strength Cond Res 2008;22(4):1371-4.

13. Wilcock IM, Whatman C, Harris N, Keogh JW. Vibration training: could it enhance the strength, power, or speed of athletes? J Strength Cond Res 2009;23(2):593-03.

14. McBride JM, Triplett-McBride T, Davie A, Newton RU. The effect of heavy-vs. light-load jump squats on the development of strength, power, and speed. J Strength Cond Res 2002;16(1):75-82.

15. Harris GR, Stone MH, O'Bryant HS, Proulx CM, Johnson RL. Short-term performance effects of high power, high force, or combined weight-training methods. J Strength Cond Res 2000;14(1):14-20.

16. Kuehl RO. Design of experiments: statistical principles of research design and analysis. London: Duxbury-Thomson Learning, 2000, pp. 666p.

17. Brown LE, Weir JP. ASEP procedures recommendation I: accurate assessment of muscular strength and power. JEPonline 2001;4(3):1-21.

18. Bosco C, Belli A, Astrua M, Tihanyi J, Pozzo R, Kellis S, et al. A dynamometer for evaluation of dynamic muscle work. Eur J Appl Physiol Occup Physiol 1995;70(5):379-86.

19. Rehn B, Lidstrom J, Skoglund J, Lindstrom B. Effects on leg muscular performance from whole-body vibration exercise: a systematic review. Scand J Med Sci Sports 2007;17(1):2-11.

20. Mileva KN, Naleem AA, Biswas SK, Marwood S, Bowtell JL. Acute effects of a vibration-like stimulus during knee extension exercise. Med Sci Sports Exerc 2006;38(7):1317-28.
21. Armstrong WJ, Nestle HN, Grinnell DC, Cole LD, Van Gilder EL, Warren GS, et al. The acute effect of whole-body vibration on the hoffmann reflex. J Strength Cond Res 2008;22(2):471-6.

22. Avela J, Komi PV. Interaction between muscle stiffness and stretch reflex sensitivity after long-term stretch-shortening cycle exercise. Muscle Nerve 1998;21(9):1224-7.

23. Avela J, Komi PV. Reduced stretch reflex sensitivity and muscle stiffness after long-lasting stretch-shortening cycle exercise in humans. Eur J Appl Physiol Occup Physiol 1998;78(5):403-10.

24. Horita T, Komi PV, Nicol C, Kyrolainen H. Stretch shortening cycle fatigue: interactions among joint stiffness, reflex, and muscle mechanical performance in the drop jump [corrected]. Eur J Appl Physiol Occup Physiol 1996;73(5):393-03.

25. Rittweger J, Beller G, Felsenberg D. Acute physiological effects of exhaustive whole-body vibration exercise in man. Clin Physiol 2000;20(2):134-42.

26. Rassier DE, Macintosh BR. Coexistence of potentiation and fatigue in skeletal muscle. Braz J Med Biol Res 2000;33(5):499-08.

27. Roelants M, Verschueren SM, Delecluse C, Levin O, Stijnen V. Whole-Body-Vibration-Induced Increase in Leg Muscle Activity During Different Squat Exercises. J Strength Cond Res 2006;20(1):124-9.

28. Wretenberg P, Feng Y, Lindberg F, Arborelius UP. Joint moments of force and quadriceps muscle activity during squatting exercise. Scand J Med Sci Sports 1993;(3):244-50.
Endereço para correspondência
Leonardo Lamas
Av. Prof. Melo de Moraes, 62.
Cidade Universitária
CEP-055808-030 São Paulo - SP - Brasil
E-mail - leonardolamas@yahoo.com.br 